\title{
Treatment of an Unyielding Central Vein Stenosis using Valvuloplasty Balloon in a Young Female Presenting with Ineffective Hemodialysis
}

\author{
Anupam Bhambhani ${ }^{1 \odot} \quad$ Amey Joshi ${ }^{1}$ \\ ${ }^{1}$ Vydehi Institute of Medical Sciences and Research Centre, \\ Whitefield, Bangalore, India \\ Ind J Car Dis Wom 2021;6:191-193.
}

\begin{abstract}
Keywords

- brachiocephalic vein stenosis

- Central vein stenosis

- Innominate vein stenosis

- valvuloplasty balloon

- venoplasty
\end{abstract}

\author{
Address for correspondence Anupam Bhambhani, M.D., D.M., \\ F.E.S.C., Associate Professor, Department of Cardiology, Vydehi \\ Institute of Medical Sciences and Research Centre, Whitefield, \\ Bangalore, India 560066 (e-mail: anupam.bhambhani@yahoo.in).
}

\section{Introduction}

Central vein stenosis (CVS) is commonly encountered in patients on maintenance hemodialysis (MHD). It eventuates from chronic endothelial injury that occurs in response to the long indwelling vascular catheters as well as the rheological effects of hyperdynamic blood flow across surgically created arteriovenous fistula (AVF).

Percutaneous transluminal balloon venoplasty (PTBV) with or without stent implantation is the treatment of choice for CVS. ${ }^{1}$ Frequently, the CVS lesions are associated with severe hypertrophy and fibrosis, since the veins ipsilateral to the AVF are persistently exposed to arterial pressure. These factors result in resistance to dilatation and high-immediate recoil. We report here an unconventional use of valvuloplasty balloon for the effective treatment of a critical innominate vein stenosis, where the lesion was resistant to dilatation with conventional balloon.

\section{Case Report}

A 40-year-old hypertensive, nondiabetic lady, with end-stage renal disease (ESRD) and on MHD via AVF created between left brachial artery and cephalic vein, presented with progressive swelling of the left upper limb and face. Doppler interrogation of the left arm revealed left innominate vein
DOI https://doi.org/ 10.1055/s-0041-1736440. ISSN 2455-7854. (c) 2021. Women in Cardiology and Related Sciences. All rights reserved.

This is an open access article published by Thieme under the terms of the Creative Commons Attribution-NonDerivative-NonCommercial-License, permitting copying and reproduction so long as the original work is given appropriate credit. Contents may not be used for commercial purposes, or adapted, remixed, transformed or built upon. (https://creativecommons.org/ licenses/by-nc-nd/4.0/)

Thieme Medical and Scientific Publishers Pvt. Ltd., A-12, 2nd Floor, Sector 2, Noida-201301 UP, India 
stenosis and patent AVF. She was referred to us for further management. There was history of MHD through right internal jugular vein (IJV) access, that is, contralateral to the AVF, for six weeks during AVF maturation period.

Clinically, there was gross edema involving left arm and left half of the face, with tortuous and dilated neck veins. There was no history of temporary catheter insertion into the affected veins.

A superior venacavogram, done via right femoral vein access, revealed severe stenosis of the left innominate vein and extensive venous collaterals (-Fig. 1 A, - Video 1).

\section{Video 1}

Superior-venacavogram showing severe stenosis in left Innominate vein and extensive venous collaterals. Online content including video sequences viewable at: https://www.thieme-connect.com/products/ ejournals/html/10.1055/s-0041-1736440.

We decided to treat the stenosis percutaneously. Written informed consent was obtained from the patient. The stenosis was crossed using a J-tipped 0.035-inch, 150 -cm Glidewire (Terumo Interventional Systems, Japan) loaded into a $5 \mathrm{~F}$ Multipurpose-A1 diagnostic catheter (Boston Scientific, USA). After exchanging the Glidewire for a stiffer 0.035inch, $260 \mathrm{~cm}$ PTFE-coated guidewire, balloon dilatation was attempted several times using a small diameter $(5 \times 80 \mathrm{~mm})$ peripheral balloon (Armada35 PTA catheter, Abbott, USA), so that space could be created for the passage of larger diameter balloons; however, the lesion recoiled immediately after each dilatation, leaving no effective lumen enlargement. It was challenging to advance a bulkier cutting balloon across the lesion, due to the resistance from tight stenosis and the acute angulation between superior vena cava (SVC) and innominate vein. After repeated unsuccessful attempts, we decided to switch to a balloon used for percutaneous transluminal mitral commissurotomy (PTMC).

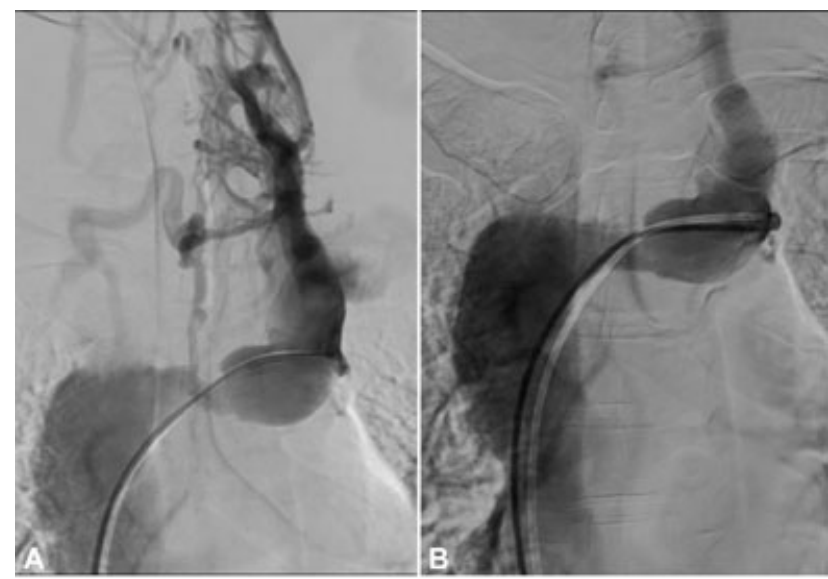

Fig. 1 (A). Severe stenosis in left innominate vein with extensive collaterals; (B). Innominate vein after dilatation with valvuloplasty balloon (note the disappearance of venous collaterals).

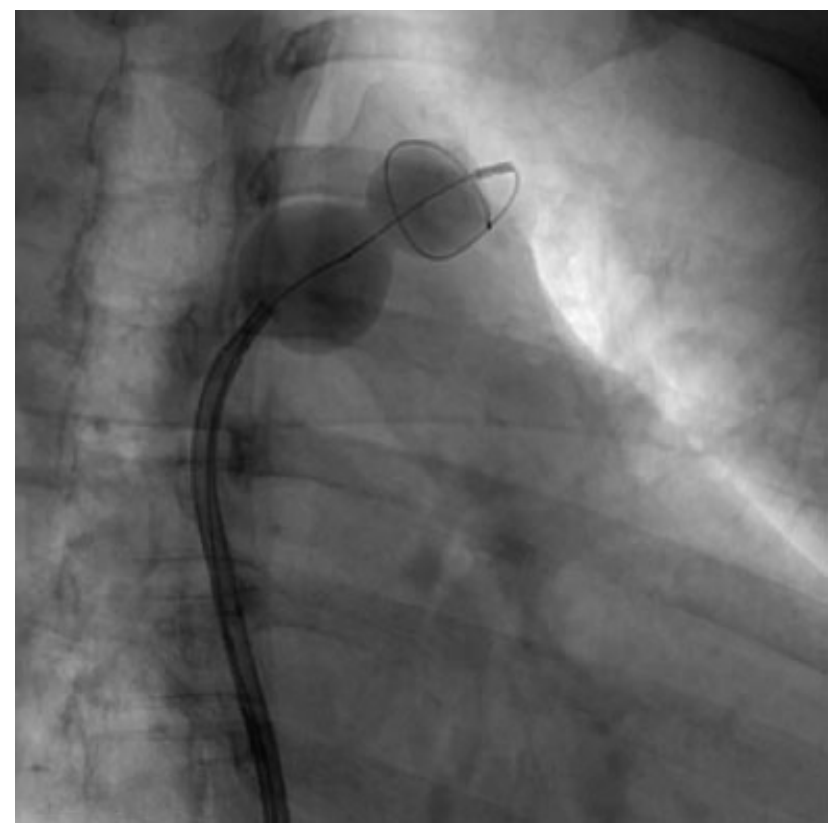

Fig. 2 Innominate vein stenosis being dilated with the help of valvuloplasty balloon.

Since the affected innominate vein was grossly dilated, the balloon inflation diameter was decided, corresponding to the contralateral innominate vein diameter. After exchanging the 0.035 -inch wire for pigtail-spring guidewire (-Fig. 2) through MP-A1 catheter, the PTMC balloon, slenderized on its stretching tube, was advanced and positioned across the stenosis. Following three dilatations (-Fig. 2), each lasting 10 minutes, the lesion yielded without recoil (-Fig. 1 B). There was significant angiographic improvement with no residual gradient, and the collaterals regressed immediately (-Fig. 1 B, - Video 2). On the following day, facial and arm swelling were found reduced. The postprocedure period was uneventful.

\section{Video 2}

Superior-venacavogram after balloon dilatation, showing angiographic improvement and regression of collaterals. Online content including video sequences viewable at: https://www.thieme-connect.com/ products/ejournals/html/10.1055/s-0041-1736440.

\section{Discussion}

The PTMC balloon was originally conceptualized by the Japanese genius Kanji Inoue for mitral valvuloplasty. In the present case, where stenosis was recoiling despite repeatedly dilating with conventional balloon, PTMC balloon helped in achieving procedural success. The dilatation in this case could be attributed to some quintessential features of the PTMC balloon: a) unlike bulky cutting/scoring balloons, it could be slenderized on its stretching tube and smoothly advanced across the challengingly acute angle between 
superior vena cava and brachiocephalic vein (-Fig. 1 A), despite the resistance imposed by critical stenosis; b) the pigtail-tip guidewire offered very good support, despite short stationing space available distal to the stenosis; c) due to its unique texture, the PTMC balloon could exert stronger force on the hypertrophied and fibrosed stenotic wall; and d) since the PTMC balloon acquires an hour-glass shape when it expands, it did not slip (melon-seed) while dilating the tight, fibrotic stenosis.

Another advantage offered by the PTMC balloon is that a single balloon provides a range of expansion diameters, allowing progressive and safe dilatations, while precluding the need for repeated balloon exchanges.

CVS often leads to inadequate hemodialysis due to fistula failure or increased venous back pressure. Various mechanisms proposed to explain the occurrence of CVS converge on to the endothelial denudation occurring secondary either to the rheological damage resulting from high velocity blood flow through AVF or to the chronic abrasion caused by venous catheters. This, in turn, results in thrombin generation, platelet activation, and initiation of an inflammatory response that culminates in intimal hyperplasia, fibrosis and, finally, stenosis at the site of venous injury. ${ }^{2}$

CVS is conventionally managed by balloon dilatation, followed by stenting in some cases. In a large study comparing venoplasty with or without stent implantation, the patency rates were not significantly different with either of the two strategies. ${ }^{1}$

To the best of our knowledge, use of this balloon has not been reported for treating CVS. The balloon dilatation was effective and safe, and postoperative recovery was uneventful with substantial improvement in symptoms.

\section{Conclusion}

Venoplasty remains the treatment of choice for CVS. In case of poor immediate response to PTV due to the acute recoil, use of PTMC balloon may be considered as a safe and effective alternative.

\section{Conflict of Interest}

The authors declare that there are no conflicts of interest concerning this case study.

\section{References}

1 Kim YC, Won JY, Choi SY, et al. Percutaneous treatment of central venous stenosis in hemodialysis patients: long-term outcomes. Cardiovasc Intervent Radiol 2009;32(02):271-278

2 Skandalou EI, Apostolidou-Kiouti FD, Minasidis ID, Skandalos IK. Left Brachiocephalic vein stenosis due to the insertion of a temporal right subclavian hemodialysis catheter. Case Rep Vasc Med 2017;2017:9524739 